\title{
Painful bruising syndrome: A case report
}

\section{Amina Aounallah ${ }^{1,2}$, Ines Lahouel ${ }^{1,2}$, Safa Belghali ${ }^{1,3}$, Asma Ben Romdhane ${ }^{1,4}$, Najet Ghariani ${ }^{1,2}$, Colandane Belajouza ${ }^{1,2}$, Mohamed Denguezli ${ }^{1,2}$, Rafiaa Nouira ${ }^{1,2}$}

\author{
${ }^{1}$ Sousse University, Sousse, Tunisia, ${ }^{2}$ Department of Dermatology, Farhat Hached Hospital, Sousse, Tunisia, ${ }^{3}$ Department \\ of Rhumatology, Farhat Hached Hospital, Sousse, Tunisia, ${ }^{4}$ Department of Psychiatry, Farhat Hached Hospital, Sousse,
} Tunisia

Corresponding author: Dr. Ines Lahouel, E-mail: Lahouel.iness@gmail.com

\begin{abstract}
Painful bruising syndrome or Gardner-Diamond syndrome is characterized by an auto erythrocyte sensitization syndrome. We report, here a new case of painful bruising syndrome in a woman with no psychiatric disorders. A 29-year-old female patient presented with complaints of painful bruising over the lower extremities of three years duration. She had a personal history of a severe rheumatoid arthritis. Dermatological examination revealed multiple erythematous, nonedematous and ecchymotic macules over the legs. A psychiatric examination was normal. Complete blood count, prothrombin time and liver function tests were normal. Intracutaneous sensitivity test confirmed Painful bruising syndrome.
\end{abstract}

Key words: Painful bruising syndrome; Gardner-diamond syndrome; Auto erythrocyte sensitization

\section{INTRODUCTION}

Painful bruising syndrome (PBS) is also known as Gardner-Diamond syndrome, auto erythrocyte sensitization syndrome or psychogenic purpura. It is a rare autoimmune vasculopathy characterized by painful ecchymotic lesions which mostly recur after emotional stress $[1,2]$. This syndrome is frequently observed in young women with psychiatric disorders [2]. PBS occurring in a psychiatrically normal person has rarely been reported. We report here a case of this condition in a woman with no psychiatric disorders.

\section{CASE REPORT}

A 29-year-old female patient presented with complaints of painful bruising over the lower extremities of three years duration. Lesions occurred at intervals of 8 weeks. There was no preceding or concurrent history of trauma, infection, psychiatric illness or bleeding from natural orifices. She had a personal history of a severe rheumatoid arthritis diagnosed in 2007. She was treated initially with methotrexate and corticosteroids, then associated with tocilizumab since 2 years. The patient reported that her lesions increased with stress and regressed spontaneously. Lesions were nonpruritic and disappeared in a week or so, leaving a bruiselike discoloration. On physical examination, her vital signs were stable. Dermatological examination revealed multiple erythematous, nonedematous and ecchymotic macules over the legs (Fig. 1). Mucosa showed no evidence of purpuric lesions. General, systemic and psychiatric examinations were normal. Skin biopsy showed dermal extravasation of red blood cells (RBC) around blood vessels without lymphohistiocytic infiltrate (Fig. 2). Complete blood count, prothrombin time and liver function tests were normal. An intracutaneous sensitivity test was done. $0.5 \mathrm{ml}$ of the patient's own blood as a source of RBCs was taken and injected into her forearm. After 24 hours, a rounded area of ecchymosis of diameter $6 \mathrm{~cm}$ was seen in the area of injection, confirming the diagnosis of PBS.

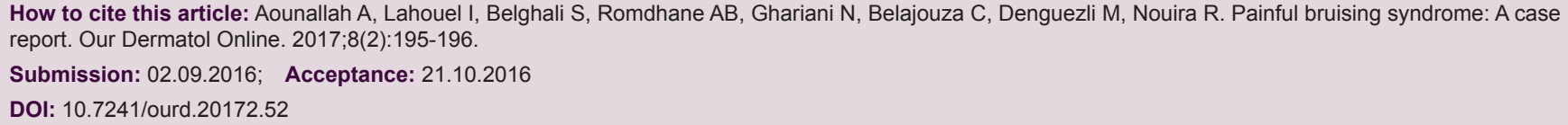




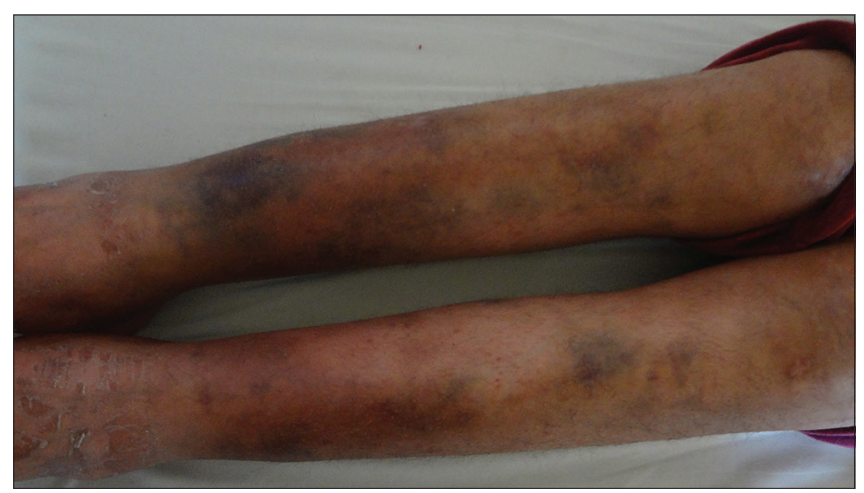

Figure 1: Multiple ecchymotic macules over the legs.

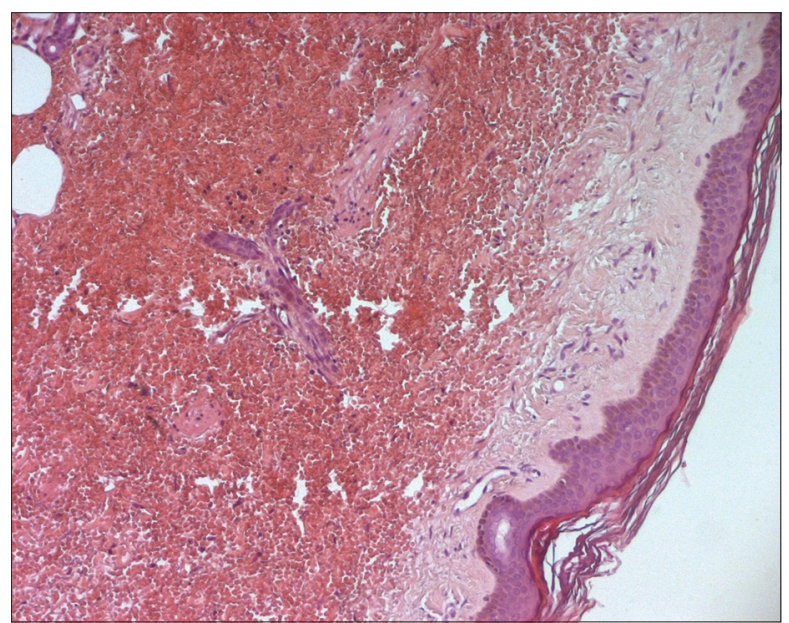

Figure 2: Dermal extravasation of red blood cells around blood vessels without lymphohistiocytic infiltrate (HE:G x100).

\section{DISCUSSION}

Painful bruising syndrome was first described by Gardner and Diamond in 1955 [3]. It is a rare syndrome characterized by spontaneous, painful inflammatory ecchymoses which commonly affect women $[4,5]$.

The etiological mechanism is not fully known. Gardner and Diamond believed that the syndrome was due to auto sensitization [3]. Recently, it has been suggested that PBS is caused by a dysfunction of neurotransmitters in the neuro-immuno-cutaneous system [6].

Many psychiatric disorders including mood and personality disorders may be observed [2]. PBS has been rarely reported in a psychiatrically normal person [1]. In the presented case, the patient experienced chronic stress due to the severity of her disease. Nevertheless, psychiatric examination hadn't revealed any psychological disorder.

Bruises are frequently located on the extremities [2]. There is usually a prodrome of warmth and pain at the bruising site, or systemic symptoms such as a headache, nausea, paresthesia, syncope, blurred vision, arthralgia, myalgia [2-5]. Mucosal bleedings may be observed [2]. Our patient hadn't any associated symptoms.

PBS may progress with recurrent attacks, as it is in our case. The intervals between attacks are variable (months or years). Painful ecchymoses usually improve in two weeks [2].

Skin biopsy shows extravasated red blood cells but no evidence of vasculitis [3]. Responses to the intra-cutaneous injection of erythrocytes are variable [3-4]. Gardner and Diamond first described cases with painful ecchymoses reproducible after injection of their own RBCs [3] similarly to our patient's case.

Many treatments have been tried, but the psychological treatment seems to be the most efficient [7].

\section{CONCLUSION}

PBS is a rare disease which is difficult to diagnose. It is frequently observed in young adult women with psychiatric disorders; however, it may occur in a psychiatrically normal person.

\section{REFERENCES}

1. Chatterjee M, Jaiswal AK. Painful bruising syndrome. Indian J Dermatol Venereol Leprol. 2002;68:347-8.

2. Ratnoff OD. Psychogenic purpura (auto erythrocyte sensitization): An unsolved dilemma. Am J Med. 1989;87:16N-21N.

3. Gardner FH, Diamond LK. Auto erythrocyte sensitization. A form of purpura producing painful bruising following out sensitizotion to red cells in certain women. Blood. 1955;10:675.

4. Sarkar S, Ghosh SK, Bandyopadhyay D, Nath S. Psychogenic purpura. Indian J Psychiatry. 2013;55:192-4.

5. Meeder R, Bannister S. Gardner-Diamond syndrome: Difficulties in the management of patients with unexplained medical symptoms. Paediatr Child Health. 2006;11:416-9.

6. Brazzini B, Ghersetich I, Hercogova J, Lotti T. The neuro-immunocutaneous-endocrine network: Relationship between mind and skin. Dermatol Ther. 2003;16:123-31.

7. Karakaş Z, Karaman S, Avcı B, Ünüvar A, Öztürk G, Anak S, et al. A disease difficult to diagnose: Gardner-Diamond syndrome accompanied by platelet dysfunction. Turk Pediatri Ars. 2014;49:250-3.

Copyright by Amina Aounallah, et al. This is an open-access article distributed under the terms of the Creative Commons Attribution License, which permits unrestricted use, distribution, and reproduction in any medium,provided the original author and source are credited.

Source of Support: Nil, Conflict of Interest: None declared. 